\title{
Quality of Anaesthesiology Care in Postoperative Patients of Outpatient Surgery with Fast Track Protocol
}

\author{
Mariel Andrea Del Rio Parra ${ }^{1}$, Luis Ángel Medina Andrade ${ }^{2 *}$, David Alejandro Jurado \\ Mendoza $^{1}$, Salvador Guadarrama ${ }^{3}$, Oscar Contreras Martínez ${ }^{1}$, Óscar Alejandro Mora Torres ${ }^{4}$, \\ Fatima Careli Morado Manríquez ${ }^{1}$, Erick Alejandro Ramírez Valencia ${ }^{1}$, Andrew Michael \\ Sorsby Vargas ${ }^{5}$, Moisés Cherem Soffer ${ }^{5}$, Fernando Eduardo fuentes Muñoz ${ }^{5}$ \\ ${ }^{\text {I}}$ Facultad Mexicana de Medicina, Universidad La Salle México, Hospital Ángeles Metropolitano, México City, \\ México. Anesthesiology Service \\ ${ }^{2}$ Gallblader and Hernia Clinic by Luis Angel Medina Andrade M.D., in MEDICI surgical center, México City, \\ México. General Surgery Service \\ ${ }^{3}$ Centro quirúrgico MEDICI de la Ciudad de México, México City, México \\ ${ }^{4}$ Facultad Mexicana de Medicina, UNAM, Hospital Ángeles Lomas, México City, México. General Surgery \\ Service \\ ${ }^{5}$ Médico Interno de Pregrado, Universidad Anáhuac Norte, México City, México
}

*Corresponding Author: Luis Ángel Medina Andrade, Gallblader and Hernia Clinic by Luis Angel Medina Andrade M.D., in MEDICI surgical center, México City, México. General Surgery Service.

\begin{abstract}
Objective: To know the satisfaction in the quality of anesthetic care in patients during the perioperative period of outpatient surgeries developed with enhanced recovery after surgery (ERAS) protocols.

Material and Method: Observational, descriptive, and cross-sectional study carried out from July 2020 to September 2020 at Gallbladder and Hernia Clinic by Luis Angel Medina Andrade M.D., in MEDICI surgical center. A total of 113 patients who fulfilled the selection criteria were included. The Bauer patient satisfaction questionnaire approved in Spanish was applied with ten questions about possible discomfort related to anesthesia and five general satisfaction questions with anesthetic care. A value of $P<0.05$ was considered statistically significant.
\end{abstract}

Results: The anesthetic care was described by $88.3 \%$ of the subjects as very satisfied and by $11.7 \%$ as satisfied. In terms of efficacy, the individuals reported being satisfied with the absence of post-anesthetic symptoms. We found that the patients who received pre-anesthetic medication presented less thirst $(40.7 \% \mathrm{vs}$. $65.4 \%, P=0.009)$ and greater satisfaction upon awakening from anesthesia $(P=0.015)$. For quality, the most significant dissatisfaction was the presence of post-anesthetic pain.

Conclusions: Our anesthetic care was perceived with high satisfaction levels, translating into a high-quality service and good doctor-patient empathy. It is necessary to improve anesthetic risks and control post-surgery pain to maintain and improve this result.

Keywords: enhanced recovery after surgery, pain control, anesthetic care, perioperative care

\section{INTRODUCTION}

Anesthesiologist function is to make evaluations that serve as a diagnostic, prognostic, and therapeutic tool during the surgical process. [1]

The presentation of new anesthetic agents with rapid onset and short action time has introduced the concept of "Fast Track" or ERAS protocols to improve perioperative efficiency by establishing a rapid recovery from anesthesia, thus facilitating early discharge and rapid resumption of activities. [2, 3] Although these protocols have been in practice in several countries and hospitals, they are not yet widely applied in Mexico. Many surgeons prefer traditional methods instead of adopting the new evidence-based protocols. [2, 4] Despite this, there is little research dedicated to studying the quality and patient safety treated with ERAS protocols in outpatient surgery. 
Patient perception regarding anesthetic management is essential to assess hospital's quality'. [5, 6] Unfortunately, there are not enough studies to measure anesthetic care quality in outpatient surgeries with ERAS protocol in Mexico.

Health Quality was defined in 2001 by the Institute of Medicine report "Crossing the abyss of quality" as: "the degree to which health services, for individuals and populations, increase the likelihood of outcomes desired health conditions and are consistent with current professional knowledge."[4, 7] However, dissatisfaction arises if the patient feels an inconsistency between the expected care and the care provided, including anesthetic care perception. $[5,8,9]$. Although the perception is subjective, its information offers a unique perspective to evaluate non-technical aspects of medical care. [5] It must be collected using rigorous methods and a valid and reliable instrument to be useful and valid for physicians and researchers to improve anesthesiology. [5, 10]

There are few studies regarding anesthesia patient satisfaction, most are not randomized, and measurements have been made with onedimensional instruments with many biases and lacking rigor. [1, 4] The studied instruments usually explore three areas related to quality. The first is communication in the patientanesthesiologist relationship; It evaluates the information provided regarding the individual's understanding of anesthesia. The second is the quality of pain control. The third area evaluated its symptoms control in the postoperative period; Of these, the most important is pain, followed by nausea, vomiting, headache, chills, dysphonia, thirst, and dizziness, among others. $[1,4]$
This work's general objective is to determine postoperative patient satisfaction in outpatient surgeries with ERAS protocol in the gallbladder and hernia clinic within the MEDICI surgical center, to have an objective panorama to improve quality, and therefore patient safety.

\section{Material AND MethodS}

The study was carried out on the MEDICI surgical center in Mexico City, with patients from the Gallbladder and hernia clinic of Dr. Luis Angel Medina Andrade. It was carried out based on Helsinki and Geneva declarations of the World Medical Association and following the Regulation of the General Health Law on Research for Health and prior approval by the Committee Ethics of the hospital and informed consent.

An observational, descriptive, and crosssectional study was conducted in postoperative elective outpatient surgery patients at the MEDICI Surgical Center in Mexico City, CDMX.

Davis type II interventions: surgeries that do not require intensive or prolonged postoperative care, and postoperative analgesia can be performed orally.

Subjects over 18 years of age who underwent elective or emergency ambulatory surgery, under general or regional anesthesia, who had the mental capacity to approve their surgery participated. After 2 hours post-surgery, they were invited to answer our questionnaire applied face to face. For convenience, those who accepted during the period from July 1 to September 30, 2020, were included.

\subsection{Inclusion, Exclusion, and Elimination Criteria}

\begin{tabular}{|c|c|c|}
\hline Inclusion criteria & Exclusion criteria & Elimination criteria \\
\hline $\begin{array}{l}\text { Patients over } 18 \text { years of age } \\
\text { who undergo general } \\
\text { outpatient surgery. } \\
\text { - } \quad \begin{array}{l}\text { Regional, general, or } \\
\text { combined anesthesia }\end{array} \\
\text { - } \text { ASA I, II, and III. } \\
\text { - } \begin{array}{l}\text { Hospitalized patients in the } \\
\text { immediate postoperative } \\
\text { period. }\end{array}\end{array}$ & $\begin{array}{l}\text { Patients in need of a } \\
\text { postanesthetic care unit } \\
\text { (PACU). } \\
\text { Oral communication } \\
\text { disability or mental } \\
\text { incapacity to answer the } \\
\text { questionnaire adequately. } \\
\text { Refusal of the patient to } \\
\text { answer the survey. }\end{array}$ & $\begin{array}{l}\text { During the survey, patients } \\
\text { present a complication that } \\
\text { would prevent them from } \\
\text { continuing. If the survey had } \\
\text { already been completed, } \\
\text { there was any reason why } \\
\text { their responses would not be } \\
\text { valid. }\end{array}$ \\
\hline
\end{tabular}




\subsection{Study Description}

The Bauer patient satisfaction questionnaire, approved in Spanish, was used to assess the subject's perception of satisfaction with the anesthesia received. This questionnaire is validated both as a written test and an English interview since it identified more patients who reported lower levels of satisfaction and were, therefore, superior in detecting anesthetic quality. [11, 12]

This questionnaire consists of 15 questions subdivided into ten questions about possible discomfort related to anesthesia (pain, nausea) and five general satisfaction questions with anesthetic care. [12]

The questions were worded to ensure clarity as each question expresses only one concept and represents a statement designed to assess how satisfied patients are with anesthetic care. A semi-dichotomous scale (no/yes, moderate/severe) was used to answer possible discomfort with anesthesia. Regarding dissatisfaction, an asymmetric bipolar rating scale was chosen, with four response alternatives based on psychometric studies (very satisfied/satisfied / not very satisfied / not satisfied). [11] This scale allowed us to obtain an accurate patient opinion based on graded responses.

Once the patient met the exit criteria from the postanesthetic care unit (length of stay in the PACU), the Ramsay scale was applied. When the patient presented a score of 2, information about informed consent was provided; it was explained that the information would be kept anonymous and confidential, in addition to applying the survey by trained personnel and completing the necessary data based on the preanesthetic assessment, the anesthesia record, and the post-anesthetic care unit record. During the interview, any doubts were resolved without influencing the individuals' responses.

\subsection{Statistic Analysis}

A univariate analysis was carried out using frequencies and percentages for categorical variables, central tendency measures, and dispersion for continuous variables. The Kolmogorov-Smirnov test was used to assess the normal distribution of the variables. Parametric variables were reported as mean and standard deviation and non-parametric variables as median and interquartile range. The bivariate analysis for categorical variables was performed using Pearson's Chi-square test or Fisher's exact test. A value of $\mathrm{P}<0.05$ was considered statistically significant.

\section{Results}

One hundred thirteen patients who met the selection criteria were included, of which 58 were women $(52.3 \%) .68 .5 \%$ were between 18 and 60 years old, and the rest $(31.5 \%)$ were more aged than $60.26 .1 \%$ suffer from arterial hypertension and $4.5 \%$ type 2 diabetes mellitus. $57.7 \%$ had average weight, $13.5 \%$ were overweight, and $28.8 \%$ were obese (Table 1 ).

Table1. Baseline characteristics of the patients

\begin{tabular}{|l|l|}
\hline Variable & \multicolumn{2}{|l|}{} \\
\hline Gender & $53(47.7 \%)$ \\
\hline Won & $58(52.3 \%)$ \\
\hline Age & $76(68.5 \%)$ \\
\hline $\mathbf{1 8 - 6 0}$ years & $29(26.1 \%)$ \\
\hline $\mathbf{6 1 - 7 4}$ years & $6(5.4 \%)$ \\
\hline Z75 years & \multicolumn{2}{|l|}{} \\
\hline Comorbities & $5(4.5 \%)$ \\
\hline Diabetes mellitus & $29(26.1 \%)$ \\
\hline Arterial hypertension & $30(27 \%)$ \\
\hline Obesity & $73.9 \pm 13.3$ \\
\hline Weight (kg) & $1.64 \pm 0.08$ \\
\hline Height (M) & \\
\hline Nutritional Condition & $64(57.7 \%)$ \\
\hline Normal & $15(13.5 \%)$ \\
\hline Overweight & $25(22.5 \%)$ \\
\hline Obesity grade I & $7(6.3 \%)$ \\
\hline Obesity grade II & \\
\hline Background & \\
\hline
\end{tabular}


Quality of Anaesthesiology Care in Postoperative Patients of Outpatient Surgery with Fast Track Protocol

\begin{tabular}{|l|l|}
\hline Smoking & $23(20.7 \%)$ \\
\hline Alcoholism & $12(10.8 \%)$ \\
\hline Drugs & $2(1.8 \%)$ \\
\hline NVPO & $1(0.9 \%)$ \\
\hline
\end{tabular}

73.9\% presented cholecystitis, $18.9 \%$ inguinal hernia, and $8.1 \%$ umbilical hernia. Most underwent laparoscopic (93.7\%) and elective
$(95.5 \%)$ surgery. The mean surgical time was $34.1 \pm 11.7$ minutes (Table 2).

Table2. Surgical characteristics of the patients

Variable

Diagnosis

Cholecystitis

Inguinal hernia

Umbilical hernia

Surgical intervention

Laparoscopic

Open

Type of surgery

Elective

Emergency

Surgical time (min)

Most of the patients were classified as ASA I or

II $(94.6 \%)$, IPID <10 (89.3\%), and received general anesthesia (91.9\%). All patients received prophylaxis for postoperative nausea and vomiting, mainly with metoclopramide $(99.1 \%)$. Preanesthetic medication was offered

$82(73.9 \%)$

$21(18.9 \%)$

\begin{tabular}{|l|}
\hline \multicolumn{2}{|l|}{} \\
\hline $\begin{array}{l}82(73.9 \%) \\
21(18.9 \%)\end{array}$ \\
\hline $9(8.1 \%)$ \\
\hline $104(93.7 \%)$ \\
\hline $7(6.3 \%)$ \\
\hline $106(95.5 \%)$ \\
\hline $5(4.5 \%)$ \\
\hline $34.1 \pm 11.7$ \\
\hline
\end{tabular}

in $53.2 \%$. The duration of anesthesia was $49.4 \pm$ 13.3 minutes. The mean dose of fentanyl they received was $255.4 \pm 36.3 \mathrm{mg}$; the majority received sufentanil, five mcg $(25.2 \%)$ or ten mcg $(70.3 \%)$ (Table 3).

Table3. Anesthetic characteristics of the patients

\begin{tabular}{|l|l|}
\hline $\begin{array}{l}\text { Variable } \\
\text { ASA }\end{array}$ & $33(29.7 \%)$ \\
\hline I & $72(64.9 \%)$ \\
\hline II & $6(5.4 \%)$ \\
\hline III & \multicolumn{2}{l|}{} \\
\hline Airway & $88(79.3 \%)$ \\
\hline IPID $<10$ & $23(20.7 \%)$ \\
\hline IPID 11-13 & \multicolumn{2}{l|}{} \\
\hline Type of anesthesia & $102(91.9 \%)$ \\
\hline General & $5(4.5 \%)$ \\
\hline Neuroaxial & $4(3.6 \%)$ \\
\hline Sedation & \multicolumn{2}{l|}{} \\
\hline NVPO Prophylaxis & $110(99.1 \%)$ \\
\hline Metoclopramide & $1(0.9 \%)$ \\
\hline Dexamethasone & $2(1.8 \%)$ \\
\hline Ondansetron & \\
\hline Antibiotic prophylaxis & $109(98.2 \%)$ \\
\hline Ceftriaxone & $2(1.8 \%)$ \\
\hline Metronidazole & \\
\hline Pre-anesthetic medication & $59(53.2 \%)$ \\
\hline Yes & $52(46.8 \%)$ \\
\hline No & $49.4 \pm 13.3$ \\
\hline Duration of anesthesia (min) & \\
\hline Fluid requirement & $14(12.6 \%)$ \\
\hline$<300$ ml & $57(51.4 \%)$ \\
\hline $300-500$ ml & $39(35.1 \%)$ \\
\hline $500-1000$ ml & $1(0.9 \%)$ \\
\hline$>1000$ ml & $255.4 \pm 36.3$ \\
\hline Fentanyl (mcg) & \\
\hline &
\end{tabular}


Quality of Anaesthesiology Care in Postoperative Patients of Outpatient Surgery with Fast Track Protocol

\begin{tabular}{|l|l|}
\hline Sufentanil $(\mathrm{mcg})$ & \\
\hline $0 \mathrm{mcg}$ & $5(4.5 \%)$ \\
\hline $5 \mathrm{mcg}$ & $28(25.2 \%)$ \\
\hline $10 \mathrm{mcg}$ & $78(70.3 \%)$ \\
\hline
\end{tabular}

The adverse effect rate was $0.9 \%$, the time to (180-300) minutes, and two patients received early diet was 105 (90-120) minutes, and the postoperative analgesia with tramadol or time to early ambulation was 140 (120-180) paracetamol (Table 4).

minutes. The patients were discharged after 200

Table4. Postoperative outcomes of the patients

\begin{tabular}{|l|l|}
\hline Variable & \multicolumn{2}{|l|}{} \\
\hline Adverse effects & $1(0.9 \%)$ \\
\hline Yes & $110(99.1 \%)$ \\
\hline No & $105(90-120)$ \\
\hline Early oral diet (min) & $140(130-180)$ \\
\hline Early ambulation (min) & $200(180-300)$ \\
\hline Time to discharge (min) & \multicolumn{2}{|l|}{} \\
\hline Prescribed postoperative analgesia & $1(0.9 \%)$ \\
\hline Tramadol & $1(0.9 \%)$ \\
\hline Acetaminophen & Anesthesiology \\
\hline
\end{tabular}

$88.3 \%$ felt very satisfied with the information provided by the anesthesiologist and $11.7 \%$ satisfied; $64 \%$ delighted upon awakening from anesthesia and $34.2 \%$ satisfied; $71.2 \%$ delighted with postoperative analgesia and $27 \%$ satisfied; $90.1 \%$ delighted with the care provided by the
Department of Anesthesiology and $9.9 \%$ satisfied; $81.1 \%$ delighted with the treatment of postoperative nausea and vomiting and $15.3 \%$ satisfied, $2.7 \%$ not very satisfied and 0.9 unsatisfied. 99.1\% would recommend the Anesthesiology Service (Table 5).

Table5. Satisfaction with anesthesiology care

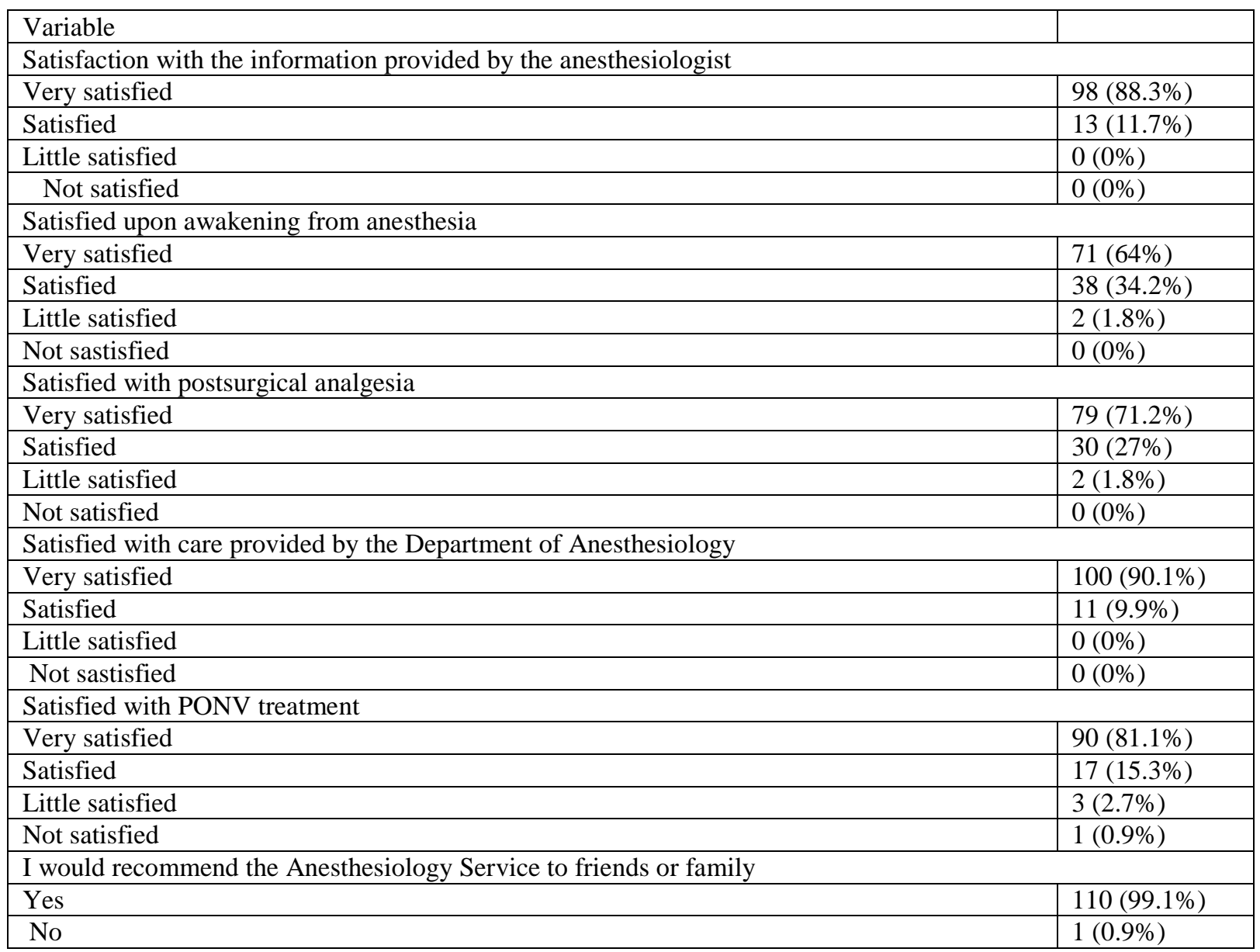


We found that the patients who received preanesthetic medication presented less thirst $(40.7 \%$ vs. $65.4 \%, \mathrm{P}=0.009)$ and greater satisfaction upon awakening from anesthesia ( $\mathrm{P}$
$=0.015)$. We did not find other differences related to symptoms or satisfaction with care (Table 6).

Table6. Relationship of pre-anesthetic medication with symptoms and satisfaction related to anesthetic care

\begin{tabular}{|c|c|c|c|}
\hline \multirow{2}{*}{ Variable } & \multicolumn{2}{|c|}{ Pre-anesthetic medication } & \multirow[t]{2}{*}{$\mathrm{P}$} \\
\hline & Sí & No & \\
\hline Prescribed postoperative analgesia & $2(3.4 \%)$ & $0(0 \%)$ & 0.497 \\
\hline Dizziness & $11(18.6 \%)$ & $15(28.8 \%)$ & 0.205 \\
\hline Surgical wound pain & $32(54.2 \%)$ & $30(57.7 \%)$ & 0.715 \\
\hline Thirst & $24(40.7 \%)$ & $34(65.4 \%)$ & 0.009 \\
\hline Hoarseness & $16(27.1 \%)$ & $15(28.8 \%)$ & 0.84 \\
\hline Sore throat & $17(28.8 \%)$ & $19(36.5 \%)$ & 0.386 \\
\hline Nausea and vomiting & $10(16.9 \%)$ & $13(25 \%)$ & 0.296 \\
\hline Cold & $10(16.9 \%)$ & $6(11.5 \%)$ & 0.418 \\
\hline Confusion or disorientation & $1(1.7 \%)$ & $2(3.8 \%)$ & 0.599 \\
\hline Pain at the anesthetic injection site & $2(3.4 \%)$ & $5(9.6 \%)$ & 0.249 \\
\hline Tremor & $6(10.2 \%)$ & $2(3.8 \%)$ & 0.279 \\
\hline \multicolumn{4}{|c|}{ Satisfaction with the information provided by the anesthesiologist } \\
\hline Very satisfied & $54(91.5 \%)$ & $44(84.6 \%)$ & \multirow[t]{2}{*}{0.259} \\
\hline Satisfied & $5(8.5 \%)$ & $8(15.4 \%)$ & \\
\hline \multicolumn{4}{|c|}{ Satisfied upon awakening from anesthesia } \\
\hline Very satisfied & $45(76.3 \%)$ & $26(50 \%)$ & \multirow[t]{3}{*}{0.015} \\
\hline Satisfied & $13(22 \%)$ & $25(48.1 \%)$ & \\
\hline Little satisfied & $1(1.7 \%)$ & $1(1.9 \%)$ & \\
\hline \multicolumn{4}{|l|}{ Satisfied with postsurgical analgesia } \\
\hline Very satisfied & $42(71.2 \%)$ & $37(71.2 \%)$ & \multirow[t]{3}{*}{0.39} \\
\hline Satisfied & $15(25.4 \%)$ & $15(28.8 \%)$ & \\
\hline Little satisfied & $2(3.4 \%)$ & $0(0 \%)$ & \\
\hline \multicolumn{4}{|c|}{ Satisfied with care provided by the Department of Anesthesiology } \\
\hline \begin{tabular}{|l|l|} 
Very satisfied \\
\end{tabular} & $55(93.2 \%)$ & $45(86.5 \%)$ & \multirow[t]{2}{*}{0.24} \\
\hline Satisfied & $4(6.8 \%)$ & $7(13.5 \%)$ & \\
\hline \multicolumn{4}{|l|}{ Satisfied with PONV treatment } \\
\hline Very satisfied & $50(84.7 \%)$ & $40(76.9 \%)$ & \multirow[t]{4}{*}{0.104} \\
\hline Satisfied & $6(10.2 \%)$ & $11(21.2 \%)$ & \\
\hline Little satisfied & $3(5.1 \%)$ & $0(0 \%)$ & \\
\hline Not sastisfied & $0(0 \%)$ & $1(1.9 \%)$ & \\
\hline \multicolumn{4}{|c|}{ I would recommend the Anesthesiology Service to friends or family } \\
\hline Yes & $58(98.3 \%)$ & $52(100 \%)$ & \multirow[t]{2}{*}{0.532} \\
\hline No & $1(1.7 \%)$ & $0(0 \%)$ & \\
\hline
\end{tabular}

\section{DISCUSSION}

It is the first study about the perception of anesthetic satisfaction carried out at the Dr. Luis Angel Medina Andrade Gallbladder and Hernia Clinic, within the MEDICI Surgical Center in Mexico City, between June to September 2020.

According to our results, we found a high perception of patients as satisfied to very satisfied. The evaluation of anesthetic satisfaction is complex because no instrument is considered the globally accepted gold standard. [13] For this reason, we used a homologation in Spanish from one of the most recognized and used in previous studies, the "Bauer Patient Satisfaction Questionnaire," since it identified more patients who reported lower degrees of satisfaction and was, therefore, superior in the detection of anesthetic quality. [11]

We established satisfaction in the two most essential dimensions considered in other studies: communication and efficacy (comprised of the ten questions about possible discomfort related to anesthesia). Regarding satisfaction in the care measured by communication, we found that, in general, people felt accompanied and informed about the anesthetic care received, corresponding to $88.3 \%$ satisfied with the information provided by the anesthesiologist and $11.7 \%$ satisfied. It should be noted that no individual rated such satisfaction as little or not satisfied. Also, no subject entered surgery 
without a prior evaluation by an anesthesiologist; For this reason, we consider high-quality communication to provide clear information on both the procedure and the risks inherent in the use of anesthetics and establishing an excellent doctor-patient relationship.

About the satisfaction of anesthetic efficacy, our instrument was mainly aimed at post-anesthetic symptoms. The individuals reported being satisfied with not having post-anesthetic symptoms. We found that the patients who received pre-anesthetic medication presented less thirst $(40.7 \%$ vs. $65.4 \%, \mathrm{P}=0.009)$ and greater satisfaction upon awakening from anesthesia $(\mathrm{P}=0.015)$. We did not find other differences related to symptoms or satisfaction with care.

In our study, sociodemographic characteristics such as age and sex did not influence the degree of satisfaction. Unlike Mira et al., there is a slight tendency to find greater satisfaction in older patients and middle and upper-income people.[13] What seems to be an agreement is that women tend to value the medical care they receive more positively, being above all satisfied with the centers' environmental characteristics. [13]

The presence of complications in this type of surgery, during or after the procedure, can influence the degree of satisfaction; In our study found that the rate of adverse effects was $0.9 \%$, a meager percentage compared to other outpatient surgery studies. $[4,13,14]$

The time to early diet was 105 (90-120) minutes, and the time to early ambulation was 140 (120-180) minutes; concepts included in ERAS protocols. The patients were discharged after 200 (180-300) minutes, and two patients received postoperative analgesia with tramadol or paracetamol. The previous indicates that a reasonable degree of analgesia helps the patient's integrated and expeditious recovery, reduces the length of stay, achieves ambulation and early diet, and especially postoperative analgesics.

Our research has several strengths. The first one is to take care of essential aspects in assessing the satisfaction, conducting the survey when the patient is more aware of their experience using a consistent questionnaire with sufficient validity, applying the survey with trained and external personnel to the study's objectives. The second was having achieved an acceptable sample size. Finally, the frequency of acceptance of participation was high since no patients met the elimination criteria, which translates to a low possibility of a selection bias.

On the other hand, several limitations must be considered for our results' extrapolation: our questionnaire covers the main domains. However, it could be extended to trans anesthetic quality, covering the points to be evaluated of ERAS protocols. Since this is the first time the Spanish-approved questionnaire has been applied, our results cannot be compared with those of other studies. Therefore, a construct validation with other instruments is necessary.

For now, we recommend exploring the validity of our instrument with other patients. However, in the absence of any other option, we consider Bauer's Spanish-approved patient satisfaction questionnaire to be used satisfactorily.

\section{CONCLusions}

We conclude that patients undergoing elective outpatient surgery at MEDICI Surgical Center in Mexico City, within the gallbladder and hernia clinic of Dr. Luis Angel Medina Andrade, have a high degree of satisfaction anesthetic management, which translates into quality service and good doctor-patient empathy.

In our study, sociodemographic characteristics such as age and sex did not influence satisfaction; these results are essential because they prioritize which data should be considered in quality management programs.

Patient perceptions are an essential component in assessing the quality of anesthesiology services. Although the perceptions are subjective, this information is essential to anesthesiologists. Patients offer a unique perspective to evaluate non-technical aspects of healthcare. However, to be helpful and valid for clinicians and researchers and to generate improvements in the practice of anesthesiology, such information must be collected using rigorous and validated methods.

The main areas for improvement are communication, increasing the information on anesthetic risks clearly and with enough empathy to generate trust, and for effectiveness, to avoid and promptly address postoperative discomfort. 


\section{REFERENCES}

[1] Moris KE, Hilal AM. Perioperative medicine: a new concept for anesthetic care. Ain-Shams J Anaesthesiol. 2017;10(1):1.

[2] Moningi S, Patki A, Padhy N, et al. Enhanced recovery after surgery: An anesthesiologist's perspective. J Anaesthesiol Clin Pharmacol. 2019;35(Suppl 1): S5.

[3] Çaparlar CÖ, Özhan MÖ, Süzer MA, et al. Fast-track anesthesia in patients undergoing outpatient laparoscopic cholecystectomy: comparison of sevoflurane with total intravenous anesthesia. J Clin Anesth. 2017;37:25-30.

[4] Fleisher LA. Quality Anesthesia. Anesthesiology. 2018 December 1;129(6):1063-9.

[5] Le May S, Hardy J-F, Taillefer M-C, Dupuis G. Patient satisfaction with anesthesia services. Can J Anaesth. 2001 Feb;48(2):153-61.

[6] Heidegger T, Saal D, Nübling M. Patient satisfaction with anesthesia - Part 1: Satisfaction as part of outcome - satisfies patients. Anesthesia. 2013 Nov;68(11):116572.

[7] Corrigan JM. Crossing the quality chasm. Build Better Deliv Syst. 2005;
[8] Fung D, Cohen MM. Measuring patient satisfaction with anesthesia care: a review of current methodology. Anesth Analg. 1998;87(5):1089-98.

[9] Bell DM, Halliburton JR, Preston JC. An evaluation of anesthesia patient satisfaction instruments. AANA J. 2004;72(3).

[10] Moonesinghe SR, Tomlinson AA. Quality improvement and revalidation: two goals, same strategy? Oxford University Press; 2011.

[11] Bauer M, Böhrer H, Aichele G, et al. Measuring patient satisfaction with anesthesia: perioperative questionnaire versus standardized face-to-face interview: Measuring patient satisfaction. Acta Anaesthesiol Scand. 2001 Jan;45(1):65-72.

[12] Barnett SF, Alagar RK, Grocott MPW, et al. Patient-Satisfaction Measures in Anesthesia. Anesthesiology. 2013 August 1;119(2):452-78.

[13] Mira JJ, Aranaz J. La satisfacción del paciente como una medida del resultado de la atención sanitaria. Med Clin Barc. 2000;114(Supl 3):2633.

[14] Lemos P, Jarrett P, Philip BK, International Association for Ambulatory Surgery. Day surgery: development and practice. London: International Association for Ambulatory Surgery;2006.

Citation: Mariel Andrea Del Rio Parra, Luis Ángel Medina Andrade, David Alejandro Jurado Mendoza, Salvador Guadarrama, Oscar Contreras Martínez, Óscar Alejandro Mora Torres, Fatima Careli Morado Manríquez, Erick Alejandro Ramírez Valencia, Andrew Michael Sorsby Vargas, Moisés Cherem Soffer, Fernando Eduardo fuentes Muñoz. "Quality of Anaesthesiology Care in Postoperative Patients of Outpatient Surgery with Fast Track Protocol”. ARC Journal of Surgery. 2021; 7(1):30-37. DOI: https://doi.org/ 10.20431/2455-572X.0701005.

Copyright: (C) 2021 Authors. This is an open-access article distributed under the terms of the Creative Commons Attribution License, which permits unrestricted use, distribution, and reproduction in any medium, provided the original author and source are credited. 